\title{
OPTIMAL LIQUIDATION OF A CALL SPREAD
}

\author{
ERIK EKSTRÖM ${ }^{1}$, CARL LINDBERG, JOHAN TYSK ${ }^{2}$, HENRIK WANNTORP $^{1}$
}

\begin{abstract}
We study the optimal liquidation strategy for a call spread in the case when the market uses an over-estimated volatility to price options. The problem is formulated as an optimal stopping problem, which we solve explicitly.
\end{abstract}

\section{INTRODUCTION}

In recent years, volatility has emerged as an asset class in its own right. As option markets are becoming increasingly liquid, new financial instruments such as variance swaps and volatility futures are introduced. However, exposure to volatility is still typically taken by various option strategies. One of the most common strategies is to take a position in an option spread. A call spread is a long position in a call option and a short position in another call on the same underlying and with the same maturity, but with a different strike price. A put spread is defined analogously. By construction, the spread has both limited profit and limited loss. As a consequence, it is considered a conservative and cautious strategy.

In any complete market model, options that trade at an implied volatility different from the true volatility allow for arbitrage opportunities. In practice, however, there is risk that cannot be hedged away due to incompleteness and market imperfections. Options are therefore often traded at an implied volatility which is larger than the true future realized volatility. We consider an investor looking to benefit from such a mispricing by investing in a call spread. If both strikes are smaller than the spot price of the underlying asset, then a large implied volatility reflects a market fear of the underlying price falling below the strikes. Consequently, if the market overestimates the volatility, then the market price of the call spread is too low. The investor would hold its position should the asset price remain above the strikes. On the other hand, if the asset price falls significantly below the strikes, then the investor believes in a smaller chance of recovery than does the market, and would therefore liquidate the position before maturity. In this paper we study the corresponding optimal liquidation strategy.

2000 Mathematics Subject Classification. Primary 91B28; Secondary 60G40.

Key words and phrases. Optimal stopping; call spread; Bachelier model.

${ }^{1}$ Supported by the Swedish Graduate School of Mathematics and Computing (FMB).

${ }^{2}$ Supported by the Swedish Research Council (VR). 
In order to set up a mathematical model for the situation described above, we make the following two assumptions.

(i) The payoff of the long and the short calls is replaced by the pay-off of a digital option.

This is a natural simplification of the problem since the digital option can be approximated arbitrarily well by a long position in $m$ numbers of call options with strike $K$ and a short position in $m$ numbers of call options with strike $K+1 / m$.

(ii) We use the Bachelier model for the underlying asset.

While the Black-Scholes model is the industry benchmark model for options, it has several drawbacks from a modeling perspective. One such drawback is that the Black-Scholes model has a constant volatility, whereas volatility typically exhibits a negative correlation with the underlying. The Bachelier model incorporates at least some aspects of this important feature, and it is analytically very tractable. A theoretical deficiency of the Bachelier model is that it allows for negative asset prices with positive probability. However, for relatively short times to expiry this objection is of no practical importance.

In the next section we show that the problem of when to optimally liquidate the call spread can be formulated as an optimal stopping problem. The literature on optimal stopping in finance is, of course, extensive. However, to the best of our knowledge, the type of problem considered here has not been treated previously even though it stems from a strategy widely used in the financial industry. The optimal liquidation boundary is shown to be of the square root type, compare Theorem 2.1 below. We also study the dependence of the size of the continuation region on model parameters. Finally, in Section 3 we investigate how the strike $K$ should be chosen.

\section{The Optimal Liquidation Problem}

As explained above, we use the Bachelier model for the underlying asset price $\tilde{X}$, and we assume for simplicity that the interest rate is zero. Thus we assume that the market models the price of the underlying under the pricing measure as

$$
d \tilde{X}_{t}=\tilde{\sigma} d \tilde{W}_{t},
$$

where $\tilde{\sigma}>0$ is a constant volatility and $\tilde{W}$ denotes a standard Brownian motion. Consider a digital option struck at $K$, i.e. an option paying the amount $g\left(\tilde{X}_{T}\right)$ at time $T$, where

$$
g(x)= \begin{cases}1 & \text { if } x \geq K \\ 0 & \text { if } x<K\end{cases}
$$

If the underlying is worth $y$ at time $t<T$, then the market value of the option is

$$
\Pi(t, y)=P\left(y+\tilde{\sigma} \tilde{W}_{T-t} \geq K\right)=\Phi\left(\frac{y-K}{\tilde{\sigma} \sqrt{T-t}}\right)
$$


where

$$
\Phi(x)=\int_{-\infty}^{x} \varphi(z) d z \quad \text { and } \quad \varphi(x)=\frac{1}{\sqrt{2 \pi}} e^{-x^{2} / 2}
$$

denote the distribution and density function of a standard normal random variable.

We consider the case of an overestimated volatility, so that the true physical asset price is in fact given by

$$
d X_{t}=\sigma d W_{t},
$$

where $0<\sigma<\tilde{\sigma}$. At any instant $t$ we may choose to liquidate our position, thereby receiving the market value $\Pi\left(t, X_{t}\right)$ of the digital option. On the other hand, if we choose to hold the option until time $T$, then we receive the amount $g\left(X_{T}\right)$. We are thus faced with the optimal stopping problem

$$
\begin{aligned}
V(t, x) & =\sup _{t \leq \tau \leq T} E_{t, x}\left[\Pi\left(\tau, X_{\tau}\right) 1_{\{\tau<T\}}+g\left(X_{T}\right) 1_{\{\tau=T\}}\right] \\
& =\sup _{t \leq \tau \leq T} E_{t, x}\left[\Phi\left(\frac{X_{\tau}-K}{\tilde{\sigma} \sqrt{T-\tau}}\right) 1_{\{\tau<T\}}+g\left(X_{T}\right) 1_{\{\tau=T\}}\right],
\end{aligned}
$$

where $\tau$ denotes a stopping time with respect to the filtration generated by $X$ and the indices denote that $X_{t}=x$.

Remark The optimal stopping problem (1) can alternatively be formulated as a regime switching problem. Indeed, the problem can be thought of as a problem of deciding a moment at which the volatility of a Brownian motion increases from $\sigma$ to $\tilde{\sigma}$, and where the objective is to maximise the probability that the Brownian motion ends up above $K$.

To solve problem (1), consider the process $Y$ given by

$$
Y_{u}=\frac{X_{u}-K}{\tilde{\sigma} \sqrt{T-u}}
$$

for $u \in[t, T)$. Note that Itô's formula yields

$$
d Y_{u}=\frac{Y_{u}}{2(T-u)} d u+\frac{\sigma}{\tilde{\sigma} \sqrt{T-u}} d W_{u},
$$

and that $\lim _{u \rightarrow T} Y_{u}$ is either $+\infty$ or $-\infty$. By performing the deterministic time-change $u=\rho_{s}$, where

$$
\rho_{s}-t=(T-t)\left(1-e^{-s}\right),
$$

we find that the process

$$
Z_{s}=Y_{\rho_{s}}, \quad s \geq 0
$$

satisfies

$$
d Z_{s}=\frac{1}{2} Z_{s} d s+\beta d W_{s}^{\prime}
$$


for some standard Brownian motion $W^{\prime}$, and $\beta=\sigma / \tilde{\sigma}<1$. The optimal stopping problem (1) satisfies

$$
V(t, x)=F\left(\frac{x-K}{\tilde{\sigma} \sqrt{T-t}}\right),
$$

where $F$ is given by

$$
F(z)=\sup _{0 \leq \tau \leq \infty} E_{z}\left[\Phi\left(Z_{\tau}\right)\right] .
$$

Here the supremum is taken over all random times which are stopping times with respect to the filtration generated by $Z$, and we use the convention that $\Phi\left(Z_{\tau}\right)=\lim _{s \rightarrow \infty} \Phi\left(Z_{s}\right)$ on the set $\{\tau=\infty\}$.

An application of Itô's formula shows that

$$
d \Phi\left(Z_{s}\right)=\frac{1}{2} \varphi\left(Z_{s}\right) Z_{s}\left(1-\beta^{2}\right) d s+\beta \varphi\left(Z_{s}\right) d W_{s}^{\prime} .
$$

Since $\beta<1$, the drift has the same sign as $Z_{s}$, and one therefore expects the optimal stopping time in the perpetual optimal stopping problem (5) to be of the form

$$
\tau_{B}=\inf \left\{t \geq 0: Z_{t} \leq B\right\}
$$

for some constant boundary $B$. Moreover, general theory for optimal stopping problems, see for example [2], suggests that the value function $F$ and the optimal stopping boundary $B$ should satisfy the free boundary problem

$$
\begin{cases}\beta^{2} F^{\prime \prime}(z)+z F^{\prime}(z)=0 & \text { if } z>B \\ F(z)=\Phi(z) & \text { if } z=B \\ F^{\prime}(z)=\varphi(z) & \text { if } z=B .\end{cases}
$$

The general solution to the ordinary differential equation $\beta^{2} F^{\prime \prime}+z F^{\prime}=0$ is given by

$$
F(z)=C \Phi\left(\frac{z}{\beta}\right)+D,
$$

and since the solution should satisfy $\lim _{z \rightarrow \infty} F(z)=1$, we must have $D=$ $1-C$. Using the conditions at the free boundary $B$, and letting

$$
\Psi(x)=1-\Phi(x),
$$

one readily verifies that the solution to $(7)$ is given by $(F, B)$, where

$$
F(z)=1-\frac{\Psi(B)}{\Psi\left(\frac{B}{\beta}\right)} \Psi\left(\frac{z}{\beta}\right) \quad z>B
$$

and $B$ satisfies the equation

$$
G(B)=G(B / \beta)
$$

where

$$
G(x)=\frac{x \varphi(x)}{\Psi(x)}
$$


Remark Equation (9) admits a unique solution, and this solution is negative. Indeed, note that

$$
\frac{\Psi^{2}(x)}{\varphi(x)} G^{\prime}(x)=\left(1-x^{2}\right) \Psi(x)+x \varphi(x)=: h(x) .
$$

It is straightforward to check that $h$ is strictly increasing for $x<0$ and strictly decreasing for $x>0$. Moreover, $h(0)=1 / 2, h(\infty)=0$ and $h(-\infty)=$ $-\infty$, so $h$ has a unique zero at some point $\hat{B}<0$. Therefore, $G$ takes its minimal value at $\hat{B}$, and it is strictly increasing for $x>\hat{B}$ and strictly decreasing for $x<\hat{B}$. The existence and uniqueness of a solution $B$ to equation (9) now follows.

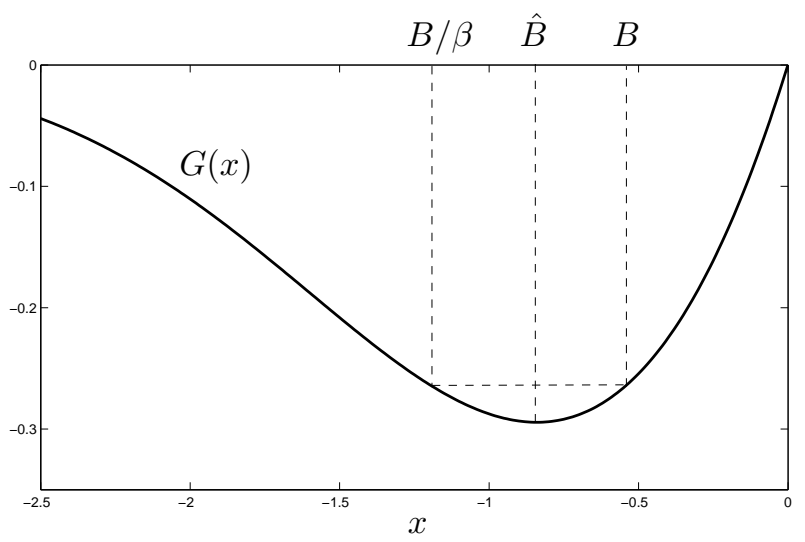

FIGURE 1. The function $G$.

Below we show that the candidate value function $F$ and the stopping time $\tau_{B}$ determined above indeed solve the optimal stopping problem (5). By the time-change (2) and relation (4), we arrive at the solution to the original problem (1).

Theorem 2.1. The value function defined in (1) is given by

$$
V(t, x)= \begin{cases}1-\frac{\Psi(B)}{\Psi\left(\frac{B}{B}\right)} \Psi\left(\frac{x-K}{\sigma \sqrt{T-t}}\right) & x>B \tilde{\sigma} \sqrt{T-t}+K \\ \Phi\left(\frac{x-K}{\tilde{\sigma} \sqrt{T-t}}\right) & x \leq B \tilde{\sigma} \sqrt{T-t}+K,\end{cases}
$$

where $B$ is the unique solution to (9). Moreover, the stopping time

$$
\tau_{*}=\inf \left\{t \leq u \leq T: X_{u} \leq B \tilde{\sigma} \sqrt{T-u}+K\right\}
$$

is optimal.

Proof. To verify that the candidate solution

$$
H(z)= \begin{cases}1-\frac{\Psi(B)}{\Psi\left(\frac{B}{\beta}\right)} \Psi\left(\frac{z}{\beta}\right) & \text { if } z>B \\ \Phi(z) & \text { if } z \leq B\end{cases}
$$


and the optimal stopping time $\tau_{B}$ indeed solve the optimal stopping problem (5), note that $H$ belongs to $C^{1}(\mathbb{R}) \cap C^{2}(\mathbb{R} \backslash\{B\})$. Moreover, since the limits $H^{\prime \prime}(B \pm)=\lim _{z \rightarrow B \pm} H^{\prime \prime}(z)$ exist and are finite, a generalised Itô formula applies (compare Problem 3.6.24 in [1]) and

$$
\begin{aligned}
H\left(Z_{t}\right)= & H(z)+\beta \int_{0}^{t} H^{\prime}\left(Z_{u}\right) d W_{u}^{\prime} \\
& +\frac{1}{2} \int_{0}^{t}\left(\beta^{2} H^{\prime \prime}\left(Z_{u}\right)+Z_{u} H^{\prime}\left(Z_{u}\right)\right) I\left(Z_{u} \neq B\right) d u \\
= & H(z)+\beta \int_{0}^{t} H^{\prime}\left(Z_{u}\right) d W_{u}^{\prime} \\
& +\frac{1}{2} \int_{0}^{t}\left(1-\beta^{2}\right) Z_{u} \varphi\left(Z_{u}\right) I\left(Z_{u}<B\right) d u \\
= & H(z)+M_{t}+\Lambda_{t},
\end{aligned}
$$

where $M_{t}$ is a martingale and $\Lambda_{t}$ is a decreasing process (recall that $B$ is negative). Since $H(z) \geq \Phi(z)$ for all $z$ it follows that

$$
\Phi\left(Z_{\tau \wedge n}\right) \leq H\left(Z_{\tau \wedge n}\right) \leq H(z)+M_{\tau \wedge n}
$$

for any stopping time $\tau$ and any nonnegative constant $n$. Therefore, the optional sampling theorem yields

$$
E_{z}\left[\Phi\left(Z_{\tau \wedge n}\right)\right] \leq H(z)+E_{z}\left[M_{\tau \wedge n}\right]=H(z) .
$$

An application of the bounded convergence theorem shows that

$$
E_{z}\left[\Phi\left(Z_{\tau}\right)\right] \leq H(z)
$$

and hence $F(z) \leq H(z)$ for all $z$.

In order to establish the reverse inequality, note that (11) yields

$$
E_{z}\left[H\left(Z_{\tau_{B} \wedge n}\right)\right]=H(z) .
$$

Letting $n \rightarrow \infty$ and using the bounded convergence theorem together with the fact that $H\left(Z_{\tau_{B}}\right)=\Phi\left(Z_{\tau_{B}}\right)$ gives

$$
E_{z}\left[\Phi\left(Z_{\tau_{B}}\right)\right]=H(z) .
$$

Thus $H(z)=F(z)$ and $\tau_{B}$ is optimal as claimed.

Remark If the investor believes that options trade at an implied volatility that is smaller than the true volatility, i.e. if $\sigma>\tilde{\sigma}$, then the investor would invest in a bull spread with a strike price $K$ that is larger than the initial asset price. A similar reasoning as above shows that the value function

$$
V(t, x)=\sup _{t \leq \tau \leq T} E_{t, x}\left[\Phi\left(\frac{X_{\tau}-K}{\tilde{\sigma} \sqrt{T-\tau}}\right) 1_{\{\tau<T\}}+g\left(X_{T}\right) 1_{\{\tau=T\}}\right]
$$

in that case is given by

$$
V(t, x)= \begin{cases}\frac{\Phi(B)}{\Phi\left(\frac{B}{\beta}\right)} \Phi\left(\frac{x-K}{\sigma \sqrt{T-t}}\right) & x<B \tilde{\sigma} \sqrt{T-t}+K \\ \Phi\left(\frac{x-K}{\tilde{\sigma} \sqrt{T-t}}\right) & x \geq B \tilde{\sigma} \sqrt{T-t}+K\end{cases}
$$


where $\beta=\sigma / \tilde{\sigma}$ and $B$ is the unique solution to

$$
G(-B)=G(-B / \beta) .
$$

We next study the dependence of $B$ on the parameter $\beta=\sigma / \tilde{\sigma}<1$.

Theorem 2.2. The optimal threshold $B$ satisfies the bounds $\hat{B}<B<$ $\beta \hat{B}$, where $\hat{B}$ is the unique value such that $h(\hat{B})=0$. Moreover, $B$ is decreasing in the parameter $\beta=\sigma / \tilde{\sigma}$. Consequently, the continuation region is increasing in the volatility $\sigma$.

Remark Numerical calculations show that $\hat{B}=-0.8399 \ldots$ One may note that the optimal stopping boundary $B$ tends to $\hat{B}$ as $\beta \rightarrow 1$. However, for $\beta=1$, the problem degenerates since $\Pi\left(t, X_{t}\right)$ is a martingale in that case.

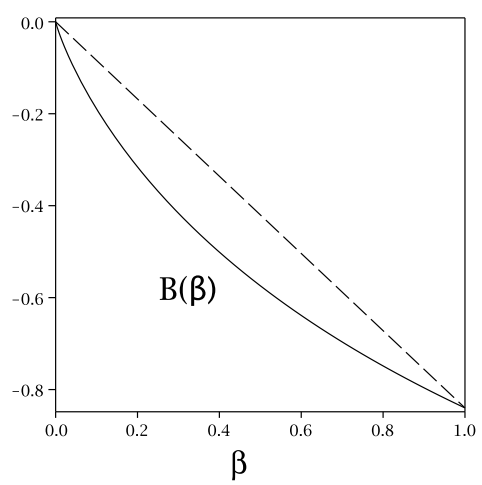

Figure 2. The picture shows the optimal threshold $B$ as a function of $\beta$ and the linear upper bound $\beta \hat{B}$.

Proof. All statements are straightforward consequences of the fact that $B$ is the unique solution to $G(B)=G(B / \beta)$, where $G$ is as in (10).

\section{The Optimal Choice of the Strike Price $K$}

In this section we ask the following question. How should the strike price $K$ be chosen in order to maximize the expected profit? To answer this, let $V(0, x ; K)$ be the value function given in Theorem 2.1, and assume that the parameters $x, T, \sigma$ and $\tilde{\sigma}$ are fixed. Let

$$
D(K)=V(0, x ; K)-\Phi\left(\frac{x-K}{\tilde{\sigma} \sqrt{T}}\right)
$$

be the difference between the option value (according to the investor) and the option price (as given by the market).

Theorem 3.1. The function $D(K)$ takes its maximal value for $K^{*}=x+$ $\tilde{\sigma} \sqrt{T} B$. 
Proof. Differentiating $D$ gives

$$
\begin{aligned}
\tilde{\sigma} \sqrt{T} D^{\prime}(K) & =\varphi\left(\frac{x-K}{\tilde{\sigma} \sqrt{T}}\right)-\frac{\Psi(B)}{\beta \Psi(B / \beta)} \varphi\left(\frac{x-K}{\sigma \sqrt{T}}\right) \\
& =\varphi\left(\frac{x-K}{\tilde{\sigma} \sqrt{T}}\right)-\frac{\varphi(B)}{\varphi(B / \beta)} \varphi\left(\frac{x-K}{\sigma \sqrt{T}}\right) \\
& =\varphi\left(\frac{x-K}{\sigma \sqrt{T}}\right)\left(\varphi\left(\frac{x-K}{\tilde{\sigma} \sqrt{T}}\right)^{1-\frac{1}{\beta^{2}}}-\varphi(B)^{1-\frac{1}{\beta^{2}}}\right)(2 \pi)^{\frac{1}{2}\left(1-\frac{1}{\beta^{2}}\right)}
\end{aligned}
$$

where we in the second equality used (9). Consequently, $D^{\prime}(K)=0$ if and only if $K=x+\tilde{\sigma} \sqrt{T} B$ or $K=x-\tilde{\sigma} \sqrt{T} B$. It is straightforward to check that $K=x+\tilde{\sigma} \sqrt{T} B$ gives the global maximum of $D$.

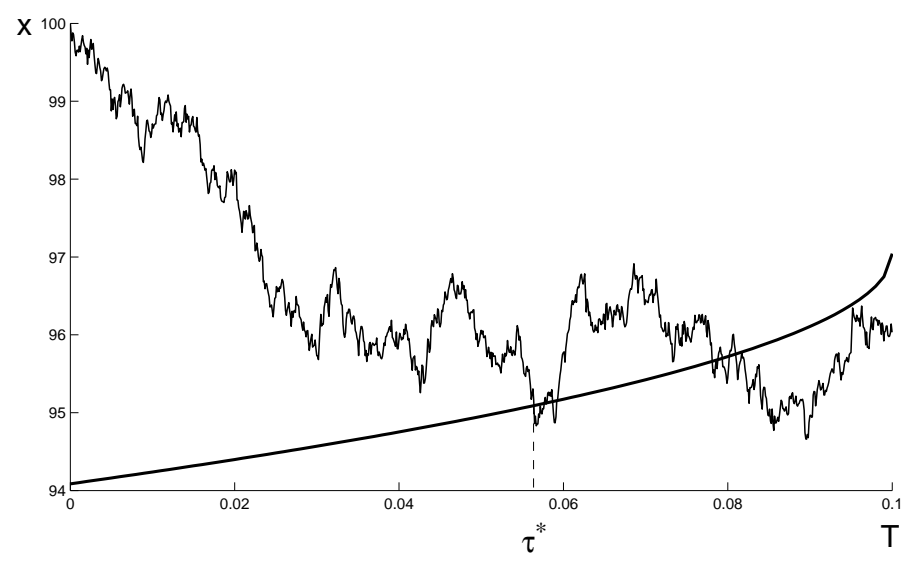

FiguRE 3 . The optimal stopping boundary. Here $x=100, T=$ $0.1, \sigma=10$ and $\tilde{\sigma}=12.5$. Consequently, $\beta=0.8, B=-0.748 \ldots$ and $K^{*}=97.04 \ldots$

\section{REFERENCES}

[1] Karatzas, I. And Shreve, S. (2000). Brownian Motion and Stochastic Calculus. 2nd ed., Springer-Verlag, New York.

[2] Peskir, G. And Shiryaev, A. (2006). Optimal stopping and free-boundary problems. Lectures in Mathematics ETH Zürich. Birkhäuser Verlag, Basel.

E. Ekström, J. Tysk and H. Wanntorp: Department of Mathematics, UppSala University, Box 480, SE-751 06 Uppsala, SWEDEN

C. Lindberg: Weavering Capital, Kungsportsavenyen 1, Box 53215, SE-400 16 GöTEBorg, SWEDEN. 